\title{
Distribuição espacial do pulgão preto em feijão de corda e cálculo do número de amostras ${ }^{1}$
}

\author{
Spatial distribution of the cowpea aphid and calculation of the sample size
}

\author{
Jefté Ferreira da Silva ${ }^{2 *}$, Ervino Bleicher ${ }^{3}$, Gleidson Vieira Marques ${ }^{4}$ e Valéria Silva ${ }^{3}$
}

\begin{abstract}
RESUMO - Objetivou-se com este trabalho estudar a dispersão espacial do pulgão preto (Aphis craccivora Koch), na cultura de feijão de corda (Vigna unguiculata (L.) Walp.) e estabelecer o número de amostras necessárias para a estimativa da população da praga para o uso em programas de Manejo Integrado de Pragas. Para isso, foram cultivados dois campos experimentais na Universidade Federal do Ceará, em Fortaleza. O primeiro campo tinha uma área de $216 \mathrm{~m}^{2}$, composta de 15 parcelas. O segundo campo tinha uma área de $576 \mathrm{~m}^{2}$ dividida em 25 parcelas. A cultivar utilizada foi a Vita 7 com plantas espaçadas em $0,25 \mathrm{~m}$ x $0,8 \mathrm{~m}$. Foram realizadas seis coletas de dados em cada campo onde foram avaliadas dez plantas por parcela. Foi contado o número de colônias de pulgão presentes em toda a planta. Os resultados obtidos nos índices de agregação utilizados indicam que a dispersão do A. craccivora no campo é do tipo agregada o que foi confirmado pelo ajuste dos dados à distribuição de frequência Binomial Negativa. Quarenta e cinco é o número de amostras adequado para a estimativa da população de A. craccivora em campos de V. unguiculata para aplicação em programas de Manejo Integrado de Pragas.
\end{abstract}

Palavras-chave: Vigna unguiculata (L) Walp. Aphis craccivora Koch. Distribuição de frequências. Manejo integrado de pragas.

\begin{abstract}
The objective of this work was to study the spatial dispersion of the cowpea aphid (Aphis craccivora Koch) in the cowpea (Vigna unguiculata (L.) Walp.) and to determine the sample size required to estimate the pest population, for use in Integrated Pest Management programs. In order to do this, two field experiments were carried out at the Federal University of Ceará in Fortaleza, Brazil. The first with an area of $216 \mathrm{~m}^{2}$, consisting of 15 plots. The second with an area of $576 \mathrm{~m}^{2}$ divided into 25 plots. The cultivar "Vita 7" was used, with plants spaced $0.25 \mathrm{~m} \times 0.8 \mathrm{~m}$ apart. Data were collected six times for each experiment, when ten plants per plot were evaluated. The total of aphid colonies present was counted for each plant. The results obtained from the clustering indices which were employed indicate that dispersion of A. craccivora in the field is of the aggregate type, which was confirmed by fitting the data to the negative binomial distribution of frequency. Forty-five is a suitable number of samples in the estimation of the population of A. craccivora in fields planted with $V$. Unguiculata, for use in integrated pest management programs.
\end{abstract}

Key words: Vigna unguiculata (L) Walp. Aphis craccivora Koch. Frequency distribution. Integrated pest management.

\footnotetext{
*Autor para correspondência

Recebido para publicação 06/09/2012; aprovado em 24/01/2014

Parte da tese de Doutorado do primeiro autor, apresentada ao Programa de Pós-Graduação em Agronomia/Fitotecnia, CCA/UFC

${ }^{2}$ Departamento de Fitotecnia/CCA, Universidade Federal do Ceará, Av. Mister Hull, s/n, Campus do Pici, Fortaleza-CE, Brasil, 60.455-760, jefteferreira@gmail.com

${ }^{3}$ Departamento de Fitotecnia/CCA, Universidade Federal do Ceará, Av. Mister Hull, s/n, Campus do Pici, Fortaleza-CE, Brasil, 60.455-760, ervino@ufc.br, valeria18ce@hotmail.com

${ }^{4}$ Universidade Federal Rural do Semi-Árido, Rua Gamaliel Martins Bezerra, s/n, Alto da Alegria, Angicos-RN, Brasil, 59.515-000, gleidson@ ufersa.edu.br
} 


\section{INTRODUÇÃO}

O pulgão preto do feijoeiro (Aphis craccivora Koch, 1854) é uma praga cosmopolita que utiliza como alimento várias espécies vegetais (RAKHSHANI et al., 2005). No feijão de corda [Vigna unguiculata (L) Walp.] os principais danos que causa é a sucção de seiva, principalmente de ramos novos, flores, vagens e folhas. Também são vetores de vírus como, por exemplo, o Cowpea aphid borne mosaic vírus (CABMV) (OBOPILE, 2006).

Atualmente, para o controle de pragas, o Manejo Integrado de Pragas (MIP) tem se tornado uma alternativa. No MIPse adotam técnicas que privilegiam o manejo da população de artrópodes que se inter-relacionam no agroecossistema (MELO et al., 2006). Assim, é fundamental o conhecimento de uma forma de amostragem rápida e eficiente da praga e de seus inimigos naturais (FERNANDES; BUSOLI; BARBOSA, 2003). Para isso, é necessário o conhecimento de sua distribuição, ou dispersão espacial.

A distribuição espacial é a forma de como os indivíduos de uma população se dispersam em seu habitat (RICKLEFS, 2003) e é fundamental para o conhecimento da etologia do inseto, estabelecimento de um plano adequado para otimização das técnicas de amostragem, definição das estratégias de controle, determinação dos danos econômicos e incorporação da dinâmica espacial dentro do modelo populacional (FARIAS; BARBOSA; BUSOLI, 2001; FERNANDES; BUSOLI; BARBOSA, 2003; RODRIGUES; FERNANDES; SANTOS, 2010).

A distribuição espacial obedece a fatores de naturezas física e biológica. No caso da natureza física, existe a hipótese de que nem todos os pontos no espaço têm a mesma probabilidade de serem ocupados. Já sobre a natureza biológica, há certos pontos onde as condições e fatores que afetam a sobrevivência são mais favoráveis que outros. Assim, locais para hibernação, postura e alimentação e o tipo de planta são fatores que podem influenciar a distribuição de organismos em uma área (GUERREIRO et al., 2005).

Adeterminação da dispersão espacial é obtida através das distribuições teóricas de frequência e do cálculo de índices de dispersão. Estes últimos, embora não descrevam matematicamente a distribuição da população estudada (ELLIOTT; KIECKHEFER; WALGENBACH, 1990) fornecem uma ideia bastante próxima da realidade quando diferentes índices fornecem resultados semelhantes. Dentre os índices mais empregados tem-se o de Morisita, Green, Razão variância/média e o índice $\mathrm{k}$ da Binomial Negativa (CESCONETTO et al., 2005; RAHMAN; FATHIPOUR; KAMALI, 2010; SEDARATIAN et al., 2010).

A dispersão espacial de uma população pode ser de três tipos: agregada (ou contagiosa) aleatória (ou ao acaso) ou uniforme (ou regular). Estes tipos geralmente seguem um modelo matemático, feita com base na relação entre a variância e a média dos dados, que descreve uma distribuição de probabilidade (MARUYAMA; BARBOSA; TOSCANO, 2006). Tais distribuições são denominadas: Binomial Negativa (Agregada), Poisson (Aleatória) e Binomial positiva (Uniforme).

Para a determinação de um número apropriado de amostras a serem tomadas é necessária uma quantidade considerável de pesquisas para encontrar um programa onde o número de amostras seja preciso e economicamente praticável (PEDIGO; RICE, 2009).

O tamanho da amostra pode ser calculado com base em um grau específico de precisão comumente mensurado pela variação relativa (VR) onde a variabilidade é expressa como uma porcentagem da relação entre o erro padrão da média e a média. Baixa VR indica grande precisão (ELLIOTT, 1977).

Assim, objetivou-se com este trabalho estudar a distribuição espacial do A. craccivora, e obter um número de amostras aplicável ao MIP em plantios de V. unguiculata.

\section{MATERIAL E MÉTODOS}

Para a realização do experimento foram instaladas duas áreas experimentais, ambas localizadas no Campus do Pici da Universidade Federal do Ceará, em Fortaleza. A semeadura da primeira área (Campo I) foi realizada no dia 11 de abril de 2011 sendo utilizada a cultivar Vita 7 por esta apresentar reconhecida susceptibilidade ao pulgão (SILVA; BLEICHER, 2010). O espaçamento foi de $0,25 \mathrm{~m}$ x $0,8 \mathrm{~m}$ numa área total do experimento de $216 \mathrm{~m}^{2}$. O espaçamento utilizado é recomendado para variedades de feijão de corda de porte prostrado e foi utilizado para facilitar a avaliação do experimento. Cada parcela tinha a dimensão de $4 \mathrm{~m}$ x 3,6 m, no total de 15, sendo estas divididas por fitas de nylon. Foi realizada uma adubação de cobertura que se constituiu da aplicação de $10 \mathrm{~kg} \mathrm{ha}^{-1}$ de nitrogênio, $10 \mathrm{~kg} \mathrm{ha}^{-1}$ de fósforo e $10 \mathrm{~kg} \mathrm{ha}^{-1}$ de potássio, provenientes dos adubos ureia, superfosfato triplo e cloreto de potássio, respectivamente.

A segunda área (Campo II) foi instalada no dia 15 de abril de 2011 também no Campus do Pici. Este estava próxima ao Campo I e por isso apresentava as mesmas características edáficas. Foram utilizados o mesmo espaçamento, cultivar e adubação de cobertura do campo experimental anterior. A área total do experimento foi de $576 \mathrm{~m}^{2}$, contendo 25 parcelas iguais também divididas por fitas de nylon.

Para tomada de dados foi contado o número de colônias de pulgão presentes em toda a planta sendo que, em cada parcela, foram avaliadas 10 plantas em uma linha 
previamente escolhida, ao acaso, antes de cada avaliação. Cada colônia era caracterizada pelo adulto cercado de suas ninfas. Caso as colônias estivessem coalescidas, cada adulto era considerado como representante de uma colônia, ou seja, era contado o número de adultos.

As avaliações do Campo I foram realizadas nas seguintes datas: 29/04/2011, 05/05/2011, 12/05/2011, 20/05/2011, 27/05/2011 e 03/06/2011. No Campo II as avaliações foram realizadas nos dias: 29/04/2011, 06/05/2011, 13/05/2011, 20/05/2011, 27/05/2011 e 03/06/2011.

Os índices de dispersão utilizados para se verificar a distribuição do pulgão preto do feijoeiro, com seus respectivos testes de afastamento da aleatoriedade, foram: Razão variância/ média (I), Índice de Morisita (I $\delta$ ), Coeficiente de Green (Cx) e Expoente $\mathrm{k}$ da distribuição binomial negativa.

As distribuições teóricas utilizadas para avaliar a distribuição espacial do pulgão preto do feijoeiro, em plantas feijão de corda, foram: Distribuição de Poisson e Distribuição Binomial Negativa.

Para se verificar o ajuste das distribuições de Poisson e Binomial Negativa utilizou-se o teste quiquadrado que consiste em comparar as frequências observadas com as frequências esperadas.

Para o cálculo do número de amostras recomendado para avaliação da população de $A$. craccivora em $V$. unguiculata foram utilizadas as avaliações dos dias 29/04/2011, 05/05/2011 e 20/05/2011 do Campo I e 06/05/2011, 13/05/2011 e 27/05/2011 do Campo II. As demais datas tiveram uma baixa infestação, apresentando muitas amostras com valor zero, possivelmente devido a ocorrência de precipitações pluviais, não fornecendo uma boa amostra para a aplicação nas fórmulas.

O número de amostras foi estimado utilizando-se a equação 1, proposta para quando a distribuição espacial do inseto é agregada (KOGAN; HERZOG, 1980), sendo:

$$
n=\left[\frac{1}{X}+\left(\frac{1}{K}\right)^{2}\right] x \frac{t^{2}}{D^{2}}
$$

onde: $k=$ expoente $k$ da binomial negativa; $X=$ número de indivíduos nas amostras; $D=$ nível de precisão estabelecido (VR) expresso como decimal; $t=$ valor do teste t para $P=\alpha=5 \%$

Outra equação utilizada para determinar o número de amostras foi considerando os níveis de precisão adequados para o manejo integrado de pragas com base na avaliação da variação relativa (VR). A variação relativa é calculada por Pedigo e Rice (2009) conforme a equação 2:

$$
V R=\frac{S_{X}}{m} \times 100
$$

sendo: $V R$ - variação relativa; $s_{x}$ - erro padrão da média; $m$ - média amostral.

Com o objetivo de obter uma quantidade de amostras aplicáveis ao MIP considerou-se uma precisão (D) de 25\%, conforme sugerido por Southwood e Henderson (2000).

Todas essas análises foram realizadas utilizando o programa para computador Microsoft Office Excel ${ }^{\circledR} 2010$.

\section{RESULTADOS E DISCUSSÃO}

A razão variância/média $(I)$ calculada apresentou valores maiores que a unidade em todas as datas de avaliação, indicando o modelo de distribuição agregada do pulgão preto do feijoeiro no campo. Essa agregação é confirmada pelos valores altamente significativos $(\mathrm{p}<0,01)$ no teste de afastamento da unidade diferenciando-o da aleatoriedade (Tabela 1).

Semelhantemente ao resultado do índice anterior, verifica-se que os resultados observados no índice de Morisita $\left(\mathrm{I}_{\delta}\right)$ foram superiores à unidade, com uma significância de $1 \%$ no afastamento da aleatoriedade, indicando, novamente, o modelo de distribuição agregada.

O índice $\mathrm{k}$ da binomial negativa, em onze das doze avaliações, indicou alta agregação do inseto $(0<\mathrm{k} \leq 2)$. Apenas no dia 05/05/2011 no Campo I foi observada agregação $(2<\mathrm{k} \leq 8)$, mas ainda caracteriza esse tipo de distribuição. Os valores do índice de Green $\left(\mathrm{C}_{\mathrm{x}}\right)$ também foram significativos, a $1 \%$ de probabilidade, para a agregação do $A$. craccivora $\left(\mathrm{C}_{\mathrm{x}}>0\right)$.

Quanto às distribuições, a de Poisson não indicou um bom ajuste para o pulgão preto demonstrando que os insetos não estão distribuídos aleatoriamente no campo. Apenas três das doze amostragens (nos dias 12/05/2011 no Campo I e 29/04/2011 e 20/05/2011 no Campo II) obtiveram um ajuste para esta distribuição (Tabela 2). Nas outras nove amostras a diferença significativa foi ao nível de $1 \%$.

Por outro lado se observa que houve um ajuste para a distribuição binomial negativa em dez das onze amostragens que tiveram graus de liberdade suficientes para a análise. Assim, nessas datas a população de pulgões apresentou arranjo agregado de seus indivíduos (Tabela 2). A avaliação no dia 27/05/2011 no Campo I foi a única amostragem que não se ajustou com a distribuição Binomial Negativa sendo a diferença no ajuste significativamente diferente a $5 \%$ de probabilidade. A amostragem do dia 12/05/2011 do Campo I não teve classes de frequências suficientes para possuir um grau de liberdade adequado para a análise.

A avaliação do campo II, nos dias 29/04/2011 e 20/05/2011, apresentou um ajuste para ambas as distribuições. Entretanto, no âmbito da estatística 
Tabela 1 - Média, variância, razão variância/média (I), índice de Morisita $\left(\mathrm{I}_{\delta}\right)$, expoente k da binomial negativa (k) e coeficiente de Green $\left(\mathrm{C}_{\mathrm{x}}\right.$ ) para distribuição espacial de Aphis craccivora Koch, 1854 em Vigna unguiculata (L.) Walp. Fortaleza, 2011

\begin{tabular}{lccrrrrc}
\hline \multirow{6}{*}{ Campo I } & Datas & Média & Variância & $\mathrm{I}$ & \multicolumn{1}{c}{$\mathrm{I}_{\delta}$} & $\mathrm{k}$ & $\mathrm{C}_{\mathrm{x}}$ \\
\cline { 2 - 7 } & $29 / 04 / 2011$ & 2,72 & 11,733 & $4,314^{* *}$ & $2,21^{* *}$ & $0,82084 \mathrm{AAG}$ & $0,00814^{* *}$ \\
& $05 / 05 / 2011$ & 4,45 & 10,666 & $2,395^{* *}$ & $1,31^{* *}$ & $3,19243 \mathrm{AG}$ & $0,00209^{* *}$ \\
& $12 / 05 / 2011$ & 0,07 & 0,103 & $1,544^{* *}$ & $10,00^{* *}$ & $0,12263 \mathrm{AAG}$ & $0,06040^{* *}$ \\
& $20 / 05 / 2011$ & 1,31 & 3,167 & $2,424^{* *}$ & $2,09^{* *}$ & $0,91775 \mathrm{AAG}$ & $0,00730^{* *}$ \\
& $27 / 05 / 2011$ & 0,69 & 3,140 & $4,529^{* *}$ & $6,11^{* *}$ & $0,19646 \mathrm{AAG}$ & $0,03426^{* *}$ \\
& $03 / 06 / 2011$ & 0,37 & 2,625 & $7,031^{* *}$ & $17,34^{* *}$ & $0,06191 \mathrm{AAG}$ & $0,10965^{* *}$ \\
\hline \multirow{3}{*}{ Campo II } & $29 / 04 / 2011$ & 0,16 & 0,239 & $1,496^{* *}$ & $4,17^{* *}$ & $0,32259 \mathrm{AAG}$ & $0,01272^{* *}$ \\
& $06 / 05 / 2011$ & 1,97 & 6,831 & $3,464^{* *}$ & $2,25^{* *}$ & $0,80040 \mathrm{AAG}$ & $0,00501^{* *}$ \\
& $13 / 05 / 2011$ & 2,68 & 17,160 & $6,412^{* *}$ & $3,02^{* *}$ & $0,49442 \mathrm{AAG}$ & $0,00810^{* *}$ \\
& $20 / 05 / 2011$ & 0,05 & 0,110 & $2,295^{* *}$ & $30,30^{* *}$ & $0,03708 \mathrm{AAG}$ & $0,11768^{* *}$ \\
& $27 / 05 / 2011$ & 3,22 & 37,741 & $11,706^{* *}$ & $4,31^{* *}$ & $0,30113 \mathrm{AAG}$ & $0,01330^{* *}$ \\
& $03 / 06 / 2011$ & 0,48 & 3,905 & $8,136^{* *}$ & $15,93^{* *}$ & $0,06727 \mathrm{AAG}$ & $0,05997^{* *}$ \\
\hline
\end{tabular}

**Teste de qui-quadrado significativo a $1 \%$ de probabilidade. ${ }^{\text {AAG: }}$ altamente agregado. ${ }^{\text {AG: }}$ Agregado

Tabela 2 - Teste de qui-quadrado de aderência das frequências observadas e esperadas pelas distribuições de Poisson e Binomial Negativa para o Aphis craccivora Koch, 1854, em Vigna unguiculata (L.) Walp. Fortaleza, 2011

\begin{tabular}{lcrccc}
\hline \multirow{2}{*}{ Data } & \multicolumn{2}{c}{ Poisson } & \multicolumn{2}{c}{ Binomial negativa } \\
\cline { 2 - 5 } & & $\chi^{2}$ & GL & $\chi^{2}$ & G.L. \\
\cline { 2 - 5 } Campo I & $65,07^{* *}$ & 27 & $17,52^{\text {ns }}$ & 26 \\
& $05 / 04 / 2011$ & $126,69^{* *}$ & 16 & $15,86^{\text {ns }}$ & 15 \\
& $12 / 05 / 2011$ & $3,12 \mathrm{~ns}$ & 1 & - & - \\
& $20 / 05 / 2011$ & $51,99^{* *}$ & 8 & $4,25^{\text {ns }}$ & 7 \\
& $27 / 05 / 2011$ & $85,74^{* *}$ & 9 & $17,07^{*}$ & 8 \\
& $03 / 06 / 2011$ & $46,91^{* *}$ & 15 & $3,97^{\text {ns }}$ & 14 \\
\hline \multirow{3}{*}{ Campo II } & $4,43^{\text {ns }}$ & 2 & $2,40^{\text {ns }}$ & 1 \\
& $29 / 04 / 2011$ & $180,51^{* *}$ & 11 & $8,27^{\text {ns }}$ & 10 \\
& $06 / 05 / 2011$ & $523,90^{* *}$ & 25 & $18,84^{\text {ns }}$ & 24 \\
& $13 / 05 / 2011$ & $4,93 \mathrm{~ns}$ & 3 & $0,41^{\text {ns }}$ & 2 \\
& $20 / 05 / 2011$ & $1726,21^{* *}$ & 41 & $36,70^{\text {ns }}$ & 40 \\
& $27 / 05 / 2011$ & $103,77^{* *}$ & 15 & $6,69^{\text {ns }}$ & 14 \\
\hline
\end{tabular}

$\chi^{2}$ : valor do qui-quadrado calculado. GL: Grau de liberdade. ns: Teste de qui-quadrado não significativo.*: Teste de qui-quadrado significativo a $5 \%$ de probabilidade. $* *$ : Teste de qui-quadrado significativo a $1 \%$ de probabilidade

ecológica, o melhor ajuste é representado pela distribuição de frequência que apresenta o menor valor de $\chi^{2}$ calculado (MELO et al., 2006). Neste caso, a Binomial Negativa apresentou o menor valor, revelando um melhor ajuste dos dados. Levando em consideração mais esta observação, no geral apenas a avaliação do dia 12/05/2011, no Campo I, se ajustou à distribuição de Poisson.
Esses resultados podem ser explicados pelo comportamento do A. craccivora no campo. Quando, na planta infestada por este pulgão, a competição por alimento e espaço aumenta, surge a forma alada do pulgão. Esses pulgões alados são voadores fracos, sendo necessário, para atingirem grandes distâncias, utilizarem a força dos ventos (KRISTOFFERSEN, 2003). Assim quando o inseto 
adquire a forma alada, ou mesmo pelo contato de uma planta com a outra, o inseto passa para as plantas vizinhas ou próximas formando "reboleiras" caracterizando a distribuição agregada. Eventualmente um inseto alado pode ser levado mais distante pelos ventos, colonizando áreas mais afastadas da sua planta de origem. Com o aumento do número de insetos na planta, ele irá novamente colonizar as plantas vizinhas e assim formar outras "reboleiras".

Os resultados dos cálculos do número de amostras, utilizando a fórmula sugerida por Kogan e Herzog (1980) (Equação 1) utilizada quando a distribuição espacial é do tipo agregada, e aplicando um nível de precisão de $25 \%$, estão na Tabela 3. Optou-se por utilizar este nível de precisão porque é um valor adequado quando a intensão é a aplicação no Manejo Integrado de Pragas (SOWTHWOOD; HENDERSON, 2000). Obteve-se uma grande amplitude nos números de amostras que variaram entre 12 e 494, sendo que três delas estão entre 80 e 85 amostras.

Aplicando a equação da variância relativa (VR) (Equação 2) até a obtenção de uma precisão menor ou igual a $25 \%$ em cada data de amostragem, foram gerados os valores contidos na Tabela 4. Observa-se, também, uma grande variação no número de amostras que varia de cinco até 45 .

Os menores números de amostras, em ambas as equações, foram obtidos no dia 05/05/2011 possivelmente devido haver uma variância baixa e uma maior média (Tabela 1) e assim maior número de insetos no campo, sendo necessário um menor número de amostras para chegar a um valor representativo da área. Já o maior número de amostras foi obtido no dia 27/05/2011. Nesta amostragem observa-se, além da segunda maior média do experimento, uma alta variância (Tabela 1) e, assim, uma maior dispersão nos dados sendo necessário um maior número de amostras, chegando até próximo de 500 (Tabela 3).

O modelo de distribuição agregado, no qual o pulgão A. craccivora se ajustou melhor, exige um maior

Tabela 3 - Estimativa do número de unidades amostrais para avaliar a população de Aphis craccivora Koch, 1854, em plantio de Vigna unguiculata (L.) Walp, obtida pela equação proposta por Kogan e Herzog (1980) com um índice de precisão de 25\%. Fortaleza, 2011

\begin{tabular}{ccc}
\hline & Datas & Número de amostras \\
\cline { 2 - 3 } Campo I & $29 / 04 / 2011$ & 81 \\
& $05 / 05 / 2011$ & 12 \\
& $20 / 05 / 2011$ & 85 \\
\hline \multirow{3}{*}{ Campo II } & $06 / 05 / 2011$ & 80 \\
& $13 / 05 / 2011$ & 195 \\
& $27 / 05 / 2011$ & 494 \\
\hline
\end{tabular}

Tabela 4 - Estimativa do número de unidades amostrais para avaliar a população de Aphis craccivora Koch, 1854, em plantio de Vigna unguiculata (L.) Walp, obtidas através de uma variância relativa (VR) menor ou igual a 25\%. Fortaleza, 2011

\begin{tabular}{cccc}
\hline & Datas & Número de amostras & VR $(\%)$ \\
\cline { 2 - 4 } Campo I & $29 / 04 / 2011$ & 15 & 20,7 \\
& $05 / 05 / 2011$ & 5 & 19,81 \\
& $20 / 05 / 2011$ & 30 & 19,32 \\
\hline \multirow{3}{*}{ Campo II } & $06 / 05 / 2011$ & 25 & 23,32 \\
& $13 / 05 / 2011$ & 35 & 19,64 \\
& $27 / 05 / 2011$ & 45 & 23,85 \\
\hline
\end{tabular}

número de unidades amostrais do que qualquer outro tipo de distribuição espacial quando na realização de um processo de amostragem (RODRIGUES; FERNANDES; SANTOS, 2010). Entretanto, se for considerado sob o ponto de vista da aplicação destes resultados em uma prática rotineira relativa à avaliação de campos submetidos ao MIP (SOUTHWOOD; HENDERSON, 2000), os resultados baseados em Kogan e Herzog (1980) superestimam os números de amostras necessárias. Por outro lado os resultados alcançados mediante o uso da equação de VR nos permite uma indicação segura de um menor número de amostras. Assim, diante destes resultados, pode-se sugerir um número de 45 amostras durante todo o ciclo da cultura tomando-se como unidade amostral toda a planta. Esse valor é semelhante ao sugerido por Sprenkel (2009) que recomenda 40 amostras para avaliação de Aphis gossypii em algodoeiro.

\section{CONCLUSÕES}

1.O pulgão preto do feijoeiro (A. craccivora) apresenta o modelo de distribuição agregada;

2. A distribuição Binomial Negativa é o modelo mais adequado para representar a distribuição do $A$. craccivora em áreas de produção de V. unguiculata;

3. Quarenta e cinco é o número de amostras adequado para a estimativa da população de $A$. craccivora em campos de $V$. unguiculata para aplicação em programas de Manejo Integrado de Pragas.

\section{REFERÊNCIAS}

CESCONETTO, A. O. et al. Distribuição espacial do dano da lagarta do cartucho do milho Spodoptera frugiperda (J.E. Smith, 1797), em Sidrolândia, Mato Grosso do Sul. Ensaios e Ciência, v. 9, n. 2, p. 305-314, 2005. 
ELLIOTT, J. M. Some methods for the statistical analysis of sample of benthic invertebrates. Ambleside: Freshwater Biological Association, 1977. 159 p.

ELLIOTT, N. C.; KIECKHEFER, R. W.; WALGENBACH, D. D. Binomial sequential sampling methods for cereal aphids in small grains. Journal of Economic Entomology, v. 83, n. 4, p. 1381-1387, 1990.

FARIAS, P. R. S.; BARBOSA, J. C.; BUSOLI, A. C. Distribuição espacial da lagarta-do-cartucho, Spodoptera frugiperda (J.E. Smith) (Lepidoptera:Noctuidae), na cultura do milho. Neotropical Entomology, v. 30, n. 4, p. 681-689, 2001.

FERNANDES, M. G.; BUSOLI, A. C.; BARBOSA, J. C. Distribuição espacial de Alabama argilacea (Hübner) (Lepidoptera: Noctuidae) em algodoeiro. Neotropical Entomology, v. 32, p. 107-115, 2003.

GUERREIRO, J. C. et al. Distribuição espacial do predador Doru luteipes (Scudder, 1876) (Dermaptera: Forficulidae) na cultura do milho. Revista Científica Eletrônica de Agronomia, v. 4, n. 7, p. 34-45, 2005.

KOGAN, M.; HERZOG, D. C. Sampling methods in soybean entomology. New York: Springer Verlag, 1980. 587 p.

KRISTOFFERSEN, L. The chemical ecology of Homoptera: from host plants to conspecific interactions. Sweden: Department of Ecology Lund University. 2003. 39 p. (Introductory paper, 147).

MARUYAMA, W. I.; BARBOSA, J. C.; TOSCANO, L. C. Distribuição espacial de Oncometopia facialis (Signoret) (Hemiptera: Cicadellidae) em pomar cítrico. Neotropical Entomology, v. 35, p. 93-100, 2006.

MELO, E. P. et al. Distribuição espacial de plantas infestadas por Spodoptera frugiperda (J.E. Smith) (Lepidoptera: Noctuidae) na cultura do milho. Neotropical Entomology, v. 35, n. 5, p. 689-697, 2006.

OBOPILE, M. Economic threshold and injury levels for control of cowpea aphid, Aphis craccivora Linnaeus
(Homoptera: Aphididae) on cowpea. African Plant Protection, v. 12, p. 111-115, 2006.

PEDIGO, L. P.; RICE, M. E. Entomology and pest management. 6. ed. New Jersey: Upper Saddle River, 2009. $784 \mathrm{p}$.

RAHMANI, H.; FATHIPOUR, Y.; KAMALI, K. Spatial distribution and seasonal activity of Panonychus ulmi (Acari: Tetranychidae) and its predator Zetzelia mali (Acari: Stigmaeidae) in apple orchards of Zanjan, Iran. Journal of Agricultural Science and Technology, v. 12, n. 2, p. 155-165, 2010.

RAKHSHANI, E. et al. Parasitoid complex (Hymenoptera, Braconidae, Aphidiinae) of Aphis craccivora Koch (Hemiptera: Aphidoidea) in Iran. Journal of Pest Science, v. 78, n. 4, p. 193-198, 2005.

RICKLEFS, R. E. A economia da natureza. 5. ed. Rio de Janeiro: Guanabara/Koogan, 2003. 470 p.

RODRIGUES, T. R.; FERNANDES, M. G.; SANTOS, H. R. Distribuição espacial de Aphis gossypii (Glover) (Hemiptera, Aphididae) e Bemisia tabaci (Gennadius) biótipo B (Hemiptera, Aleyrodidae) em algodoeiro Bt e não-Bt. Revista Brasileira de Entomologia, v. 54, n. 1, p. 136-143, 2010.

SEDARATIAN, Aet al. Population density and spatial distribution pattern of Thrips tabaci (Thysanoptera: Thripidae) on different soybean varieties. Journal of Agricultural Science and Technology, v. 12, n. 3, p. 275-288, 2010.

SILVA, J. F.; BLEICHER, E. Resistência de genótipos de feijão-de-corda ao pulgão-preto. Pesquisa Agropecuária Brasileira, v. 45, n. 10, p. 1089-1094, 2010.

SOUTHWOOD, T. R. E.; HENDERSON, P. A. Ecological methods. 3. ed. Oxford: Blackwell Science. 2000. 575 p.

SPRENKEL, R. K. Cotton pest monitoring manual for Florida. Institute of Food and Agricultural Sciences: University of Florida, 2009. Disponível em: <http://edis.ifas. ufl.edu/pdffiles/NF/NFREC2100.pdf >. Acesso em 11 ago. 2011. 